\title{
Les mystérieuses antiquités de Prosper Biardot
} (1805-1873)

The mysterious antiquities of Prosper Biardot (1805-1873)

\section{Angélique Allaire}

\section{OpenEdition}

\section{Journals}

Édition électronique

URL : http://journals.openedition.org/cel/5328

DOI : $10.4000 /$ cel. 5328

ISSN : 2262-208X

Éditeur

École du Louvre

Référence électronique

Angélique Allaire, "Les mystérieuses antiquités de Prosper Biardot (1805-1873) », Les Cahiers de l'École du Louvre [En ligne], 14 | 2019, mis en ligne le 02 décembre 2019, consulté le 05 décembre 2019. URL : http://journals.openedition.org/cel/5328; DOI : 10.4000/cel.5328

Ce document a été généré automatiquement le 5 décembre 2019.

\section{(c)}

Les Cahiers de l'École du Louvre sont mis à disposition selon les termes de la licence Creative Commons Attribution - Pas d'Utilisation Commerciale - Pas de Modification 4.0 International. 


\title{
Les mystérieuses antiquités de Prosper Biardot (1805-1873)
}

The mysterious antiquities of Prosper Biardot (1805-1873)

\author{
Angélique Allaire
}

\section{NOTE DE L'AUTEUR}

Cet article présente les résultats de recherches menées dans le cadre d'un mémoire de Master 2 à l'École du Louvre intitulé E. Prosper Biardot (1805 - 1873). Archéologue autodidacte et collectionneur. Nous tenons à remercier Corinne Jouys Barbelin, conservatrice du patrimoine au musée d'Archéologie nationale pour le soutien qu'elle a apporté à ce travail et Violaine Jeammet, conservatrice en chef du patrimoine au musée du Louvre, pour ses précieux conseils lors de la rédaction du mémoire et de cet article.

1 «Sous le pseudonyme d'E. Prosper Biardot se cache la collectionneuse anglaise Elizabeth Caroline Hamilton Gray (1800-1887), née Johnstone, épouse du théologien écossais John Hamilton Gray (1800-1867); Elle publia 2 ouvrages sous le nom de Biardot $»^{1}$. C'est ainsi que la bibliothèque numérique de l'Institut national d'histoire de l'art présente Prosper Biardot. De même, la base de données IdRef renvoie la fiche d'Elizabeth Caroline Hamilton Gray à la recherche "Prosper Biardot $»^{2}$. Cette identification erronée apparaît régulièrement dans des articles et bases de données, dont l'origine est probablement à chercher chez Wilhelm Fröhner ${ }^{3}$. L'antiquaire anglaise Elizabeth Caroline Hamilton Gray et Biardot n'ont en réalité aucun lien. S'ils partagent un intérêt pour l'archéologie, avec des fortunes diverses, ils ne se sont probablement jamais rencontrés et il s'agit de deux individus distincts. En réalité, Fröhner faisait référence à la compagne de Biardot, Amelia Giovanna O'Donovan Hamilton. Malgré le manque d'informations sur Biardot, il a été possible, à partir de documents d'archives, de reconstituer son identité et d'éclairer son parcours: les archives notariales conservées aux Archives nationales de Paris, le fonds Wilhelm Fröhner conservé à Weimar ${ }^{4}$ et la correspondance de Johann Jakob Bachofen conservée 
à la bibliothèque de l'Université de Bâle ${ }^{5}$ ont ainsi pu livrer des informations conséquentes qui ont permis de reconstituer le parcours de notre antiquaire.

\section{L'homme et l'archéologue}

2 Le récit de la vie de Prosper Biardot est celui d'une ascension sociale. Né en 1805, fils d'un riche pâtissier parisien, Biardot séjourne à Naples ${ }^{7}$ à partir de $1835^{8}$ où il rencontre Amelia Giovanna O'Donovan Hamilton, fille adoptive d'un prince de Cimitile' Celle-ci devient sa compagne et l'influence très probablement dans sa décision de constituer une large collection d'antiques sur le marché de l'art napolitain, encore très actif et fréquenté par des collectionneurs comme le marquis Campana ${ }^{10}$ ou Eugène Piot. De retour en France vers 1852, Biardot tente alors de s'insérer dans le milieu de l'archéologie. Grâce à sa collection, qu'il fait visiter, il rencontre conservateurs et savants parisiens, tels Adrien de Longpérier ${ }^{11}$ ou Fröhner ${ }^{12}$. Cependant, les faux grossiers qui composent l'orfèvrerie de sa collection qu'il tente de vendre au musée du Louvre et ses idées sur le symbolisme des terres cuites lui ferment les portes. L'époque voyait pourtant la parution des premières études scientifiques sur les figurines en terre cuite, notamment autour de la question de leur fonction, qui alimente violemment les propos pour aboutir, selon le mot de Salomon Reinach, en une "querelle des symbolistes et des réalistes $»^{13}$. Les premiers voient dans chaque figurine la figuration d'une divinité tandis que les seconds suggèrent plutôt d'y voir l'expression d'une vie quotidienne $^{14}$. Les théories de Biardot le classent sans hésiter dans le camp des symbolistes, alors qu'eux-mêmes ne se reconnaissent en rien dans cet amateur autodidacte ${ }^{15}$. C'est auprès du philologue bâlois Johann Jakob Bachofen ${ }^{16}$ que Prosper Biardot va trouver un réel soutien. C'est lui qui entre en contact avec Biardot en 1864 suite à la lecture du Symbolisme des terres cuites. Le savant apprécie les personnes qui, comme Biardot, "n'appartiennent à aucune coterie et ne sont pas des professeurs établis, mais qui, en revanche, ont passé des années sur le terrain à étudier $»^{17}$. En 1867, il publie même un ouvrage intitulé La doctrine de l'immortalité dans la théologie orphique sur les monuments funéraires de l'Antiquité: d'après un vase de Canosa en la possession de M. Prosper Biardot à Paris ${ }^{18}$ dans lequel il analyse un des vases de son ami grâce à une chromolithographie qu'il lui a fait parvenir (fig. 1). Ils correspondent jusqu'en 1872, peu avant le décès de Biardot, et ces lettres se sont révélées très précieuses pour comprendre la personnalité de notre collectionneur. 
Fig. 1

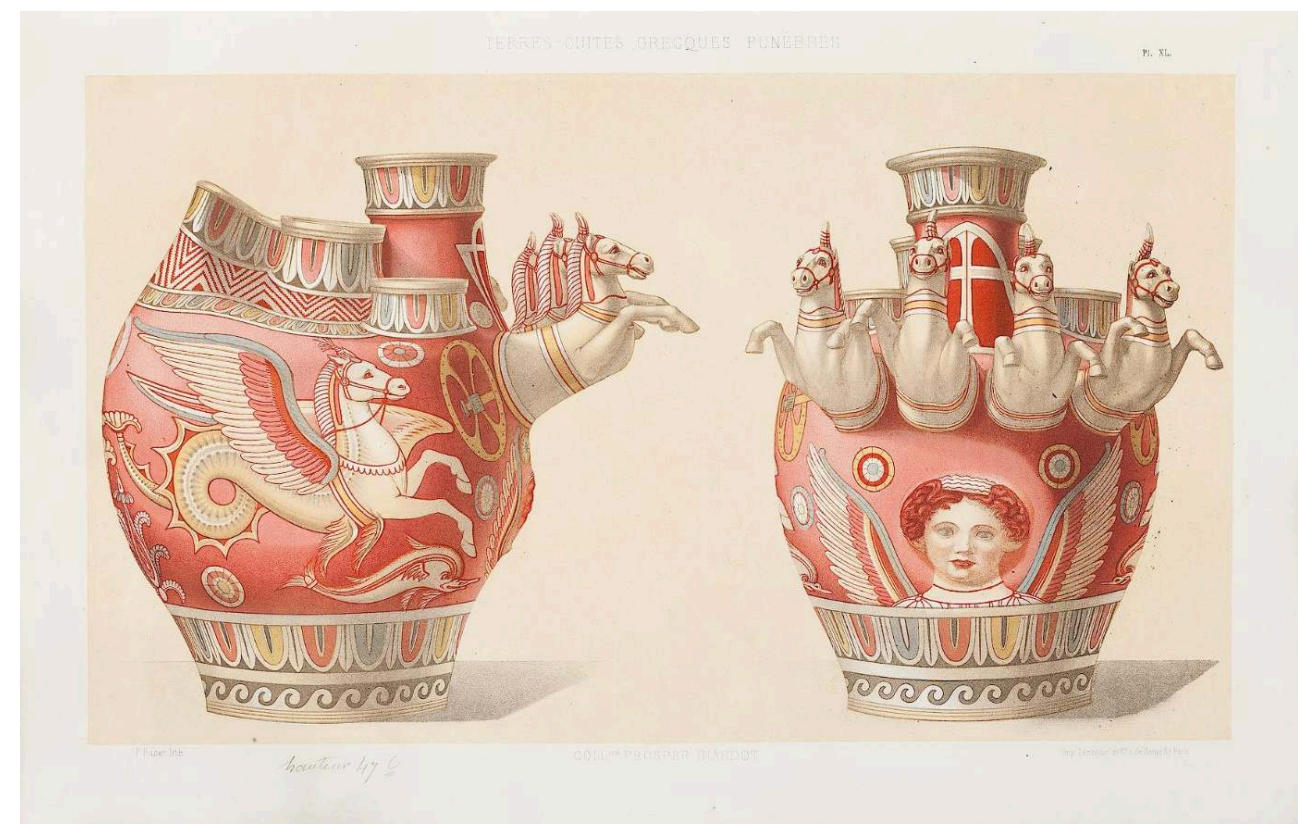

Biardot, Prosper, Atlas, planche XL : vase plastique avec protomées en forme de cheval. Heidelberg University Library

\section{Vie et destin d'une collection}

Biardot constitue sa collection sur le marché de l'art napolitain entre 1835 et 1852, encore actif et alimenté. Malheureusement, nous ne disposons pas d'un catalogue exhaustif des objets et les principales informations que nous avons retrouvées proviennent de son inventaire après décès ${ }^{19}$, de l'Atlas et d'un catalogue manuscrit d'orfèvrerie conservé à l'INHA ${ }^{20}$. Ces sources ne nous permettent pas de connaître l'évolution de la collection et les seules indications de provenance sont celles données par notre antiquaire. Sa collection se compose, d'une part, d'un ensemble d'orfèvrerie remarquable par la quantité de faux grossiers qu'il contient et, d'autre part, de terres cuites, figurines et vases plastiques, provenant majoritairement de Grande Grèce, dont un certain nombre, de l'hypogée Lagrasta I à Canosa di Puglia ${ }^{21}$. Ces derniers alors en possession du vicomte de Janzé, avant son décès en 1865, et du marquis Campana sont principalement décrits dans Les terres cuites grecques funèbres dans leur rapport avec les mystères de Bacchus ${ }^{22}$ et dans Les terres cuites grecques funèbres dans leur rapport avec les mystères de Bacchus: l'auteur a ajouté en faveur des artistes une série de figurines drapées tirées de sa collection et de l'ancien cabinet de M. le vicomte de Janzé23 de 54 planches chromolithographiées. Ils avaient, dès leur découverte, attiré l'attention des amateurs de l'époque, tels Lancelot Théodore Turpin de Crissé24 qui les qualifie de «spécimens tout exceptionnels qui manquent à nos musées ${ }^{25}$. Si des objets de l'ancienne collection du vicomte de Janzé, comme annoncé dans le titre, y sont bien représentés, on remarque aussi des ressemblances frappantes avec certains objets de la collection Campana conservés depuis 1863 au musée du Louvre. Au-delà de certaines coïncidences, puisque la technique de la coroplathie procédant par moulage permet de produire un objet en plusieurs exemplaires, ces dernières sont toutefois trop nombreuses pour n'être qu'un accident. Les planches ne précisant pas l'appartenance des objets, il semble que Biardot 
ait voulu donner de l'importance à sa collection par contamination, voire ait cherché à entretenir le flou entre ses objets et ceux de collections prestigieuses.

En revanche, de manière très moderne pour le XIX ${ }^{e}$ siècle, Biardot, dans Les terres cuites, se montre aussi sévère avec les restaurations qu'avec les contrefaçons. Les seules restaurations qu'il juge pertinentes sont celles qui visent à préserver l'intégrité d'un objet brisé : recoller des fragments ou reconstituer des petits éléments d'une figurine avec une terre qui conservera un aspect différent de la matière antique afin de pouvoir distinguer au premier coup d'œil les parties modernes. Ses préceptes peuvent se résumer ainsi : "conservez et préservez ${ }^{26}$. Pourtant ce discours contraste avec des pratiques que Biardot admet dans d'autres parties des Terres cuites. Ainsi, a-t-il luimême restauré très librement des vases de sa collection. Au sujet d'un vase représenté aux planches XLI et XLII de l'Atlas, il écrit ainsi : «Je ne dis rien de la face lunaire ou hermétique surmontée d'un génie que la planche lithographique montre à la partie postérieure du vase, parce que ces deux figures sont rapportées. À l'époque, déjà bien ancienne, où je commençais ma collection, j'avais imaginé de boucher avec ce mascaron un large trou qui me faisait de la peine, et de l'enjoliver, par surcroît, d'un génie qui ressemblait à ses voisins ${ }^{27}$ (fig. 2). Cette restauration, guère exceptionnelle pour le XIX siècle, semble pourtant chagriner Biardot qui ajoute : «Loin d'embellir mon urne, ces raccommodages lui ôtaient son caractère d'unité $\aleph^{28}$ et assure qu'il veut les supprimer. Aucun indice ne nous est parvenu quant à l'exécution de cette déclaration, qui témoigne d'une évolution dans la manière de percevoir ses antiques.

Fig. 2

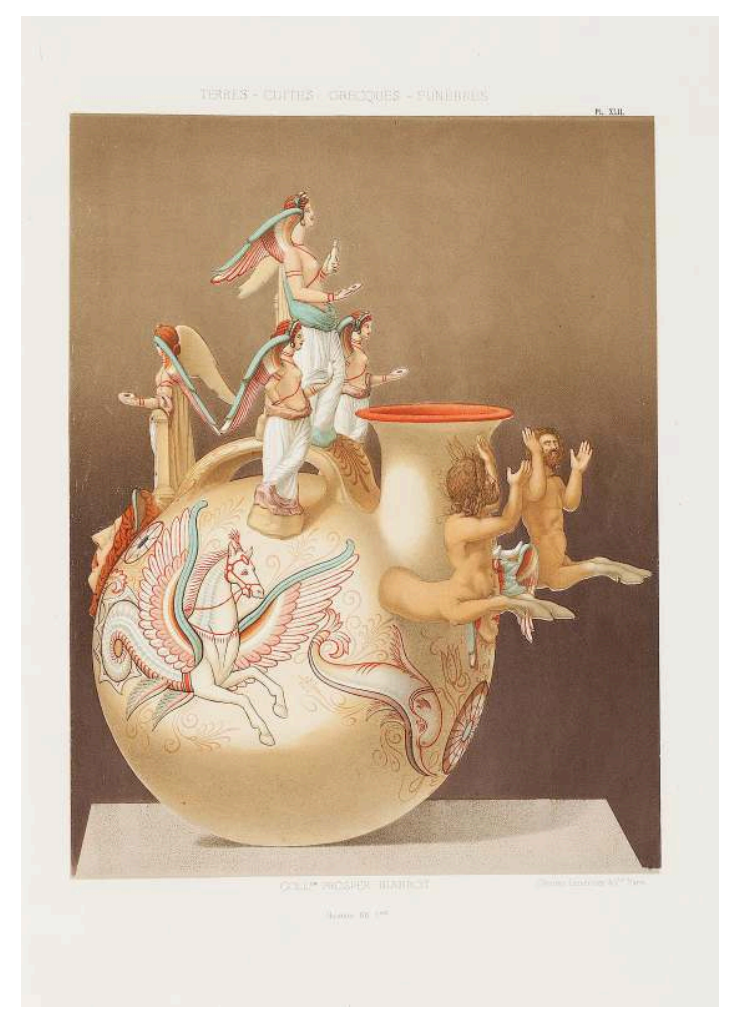

Prosper, Atlas, planche XLII : askos plastique. Heidelberg University Library

5 Ce n'est qu'après le décès de Biardot, en $1873^{29}$, que sa collection est dispersée. En février 1874, Thérèse Diebolt, sa veuve ${ }^{30}$, organise une première vente à Drouot, dont 
malheureusement le catalogue ne nous est pas parvenu. Un compte rendu publié dans L'indicateur de l'archéologue et du collectionneur déplore la faible publicité autour de cette vente et son produit décevant: moins de 3000 francs pour les 360 terres cuites réunies ${ }^{31}$. L'orfèvrerie, elle, ne trouve pas preneur. Pour s'en défaire, Diebolt fait appel à un courtier qui la cède à un intermédiaire nommé Ed. de Verdier. Ce dernier ne parvient pas à vendre la collection, prétendument rapportée de Pompéi et Herculanum et, en 1897, il attaque Diebolt en justice, en l'accusant de lui avoir sciemment vendu des contrefaçons. Au terme d'un long procès ${ }^{32}$, Verdier obtient gain de cause et l'orfèvrerie est définitivement vendue à Drouot en 1904 sous le titre "Argenterie de style antique ». Après ces ventes, malheureusement peu documentées, retrouver des objets ayant appartenu à Biardot dans des collections contemporaines semblait compromis. Pourtant il a été possible de localiser dans des musées européens certaines des terres cuites de notre antiquaire grâce au travail de Fröhner. En 1891, pour une vente à Drouot, celui-ci rédige en effet le catalogue des terres cuites antiques de la collection de Julien $\mathrm{Gré}^{3} \mathrm{u}^{33}$ dont trente-quatre sont mentionnées provenant de la collection Biardot. En retournant vers les minutes de la vente conservées aux Archives de Paris ${ }^{34}$, il a été possible de localiser certaines pièces, directement achetées par des musées, comme le musée du Louvre ${ }^{35}$, l'Altes Museum de Berlin ${ }^{36}$ ou le Nationalmuseet de Copenhague ${ }^{37}$. Une paire d'askoï ayant appartenu à Biardot est également achetée par Camille Rollin. Or le musée des beaux-arts de Lyon conserve une paire de vases identique ${ }^{38}$, qui porte des étiquettes mentionnant le nom de Gréau, et que le musée a achetée auprès de FélixBienaimé Feuardent, marchand d'art très proche de Rollin. Ces deux askoï proviennent donc très probablement de la collection de notre antiquaire. Dans un catalogue des collections du château de Gołuchów, rédigé par Fröhner ${ }^{39}$, on retrouve encore la mention de deux vases ayant appartenu à Biardot et aujourd'hui conservés, pour l'un au musée national de Varsovie ${ }^{40}$, l'autre au musée national de Cracovie ${ }^{41}$.

D'autres objets ont également été retrouvés. Ainsi un petit groupe d'Aphrodite et Pan, reproduit à la figure 2 de la planche IX, a été acheté par le sculpteur Antoine Bourdelle après être passé dans la collection Gréau ${ }^{42}$. Par ailleurs Biardot a tenté de diffuser sa collection aussi largement que possible, auprès des amateurs d'antiques mais également auprès des artistes. Il fréquente ainsi Sébastien Norblin ${ }^{43}$ avec qui il entretient probablement une relation amicale. On retrouve la trace, dans un legs de ce dernier à l'École nationale supérieure des beaux-arts de Paris en 1884, un "vase funéraire provenant des fouilles de Campanie dont l'estampage est dû à Biardot » et un «moulage en plâtre colorié reproduisant un vase de la collection Biardot $»^{44}$. Dans ce legs se trouvent aussi une "suite de moulages exécutés sur 60 figurines antiques en terre cuite " ainsi que quelques figurines de terres cuites de type tanagréen à la provenance inconnue. Malheureusement, toutes les pièces constitutives de ce legs n'ont pas été conservées et seules treize figurines sont visibles. Parmi elles, deux reprennent exactement des figures de l'Atlas. Il s'agit de la figurine MU 6247 qui correspond à la figure 1 de la planche XXV et de la statuette MU 6215 avec la figure 1 de la planche XXIX. Étant donné la proximité de notre collectionneur avec Norblin, et la ressemblance des figurines avec les représentations commandées par Biardot, il est possible que ces deux figurines de plâtre soient des tirages en plâtre de statuettes ayant appartenu à notre archéologue. Dès lors la question est ouverte : les autres figurines de plâtre sont-elles des moulages d'objets de la collection de Biardot? 
7 La collection de Biardot est donc inégalement connue en raison des sources lacunaires dont nous disposons. Sur un total de 225 objets identifiés comme ayant appartenu à Biardot il nous a été possible de n'en reconnaître que douze : les procédés de vente, par lots, et la production en série, inhérente au processus de fabrication des terres cuites, ont de fait compliqué les recherches. Quant à l'orfèvrerie, son absence de valeur historique lui a probablement barré l'entrée d'une collection privée suffisamment prestigieuse pour être documentée.

\section{De « longues recherches sur un petit coin de l'Antiquité ${ }^{45}$}

Biardot est l'auteur de deux ouvrages, Explication du symbolisme des terres cuites ${ }^{46}$ et Les terres cuites, qui vient l'approfondir, lequel est accompagné d'un Atlas qui reste un document encore consulté et cité. Publié en 1872, ce dernier vise à faire connaître et peut-être surtout à donner ses lettres de noblesse à sa collection, à laquelle ont été mêlés des objets de l'ancien cabinet du vicomte de Janzé et, comme nous l'avons suggéré plus haut, certains objets de la collection Campana du musée du Louvre. Il se compose de 54 planches colorées, que Biardot dit avoir voulues aussi fidèles à la réalité que possible. Il note ainsi "Nous avons veillé, ici comme ailleurs, à ce que le dessinateur ne corrigeât ou n'embellit pas son modèle. La fidélité est une des premières conditions à observer dans la reproduction des monuments antiques; j'ai établi cette règle générale dès le début de mon travail, et, autant qu'il a dépendu de moi, je ne m'en suis jamais écarté $»^{47}$. Il déplore cependant que les dessinateurs aient pu se laisser influencer par leur goût personnel : «malgré nos instantes recommandations de rendre son modèle avec une exactitude judaïque, l'artiste qui a dessiné nos planches a vu là un défaut qu'il a malheureusement cru devoir corriger $»^{48}$. Par ailleurs, la technique de la chromolithographie, dont il semble être l'un des derniers tenants, présente des contraintes au niveau du choix des couleurs, auxquelles notre amateur s'est également heurté. Il écrit ainsi que sujet de la planche XLVI : « La coiffure du dieu était l'emblème du fluide éthéré : sa couleur devait donc être le pourpre vif ; mais la nuance vineuse de la panse du vase s'opposant à l'emploi de cette couleur, l'artiste a dû la remplacer par le blanc, en se contentant, pour rester fidèle à la tradition, de teinter de pourpre les contours et les plis du bonnet; de cette façon le goût était sauf et le symbole avait conservé toute sa signification $»^{49}$. Au vu, non seulement de la fraîcheur des couleurs et des contraintes liées à la technique de la chromolithographie, mais aussi de l'état de conservation apparent des objets, qui apparaissent presque tous intacts sur les planches, on devine le travail de reconstitution effectué. La comparaison entre la planche XLIV et la paire d'askoï qui lui a servi de modèle, conservée au musée des beaux-arts de Lyon sous les numéros E 406-3-a et E 406-3-b, même si son état a pu s'altérer depuis le $\mathrm{XIX}^{\mathrm{e}}$ siècle, souligne aussi ce décalage entre les objets et leur représentation (fig. 3 et 4 ). 
Fig. 3

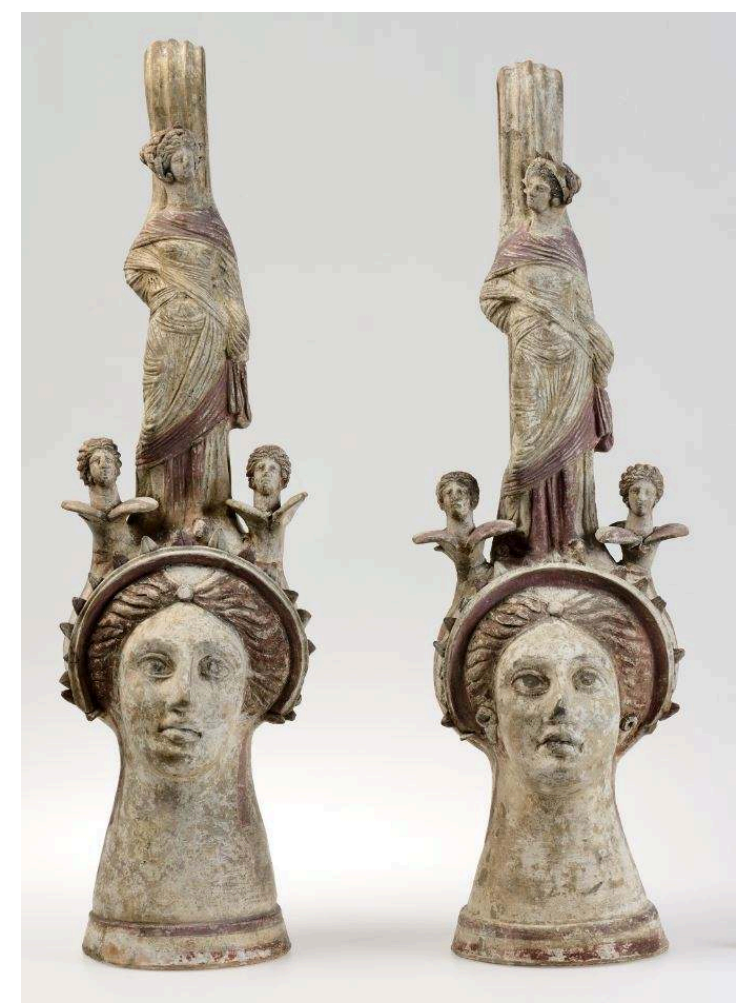

Askos, fin du Iv e siècle - début du II siècle avant J.-C., Canosa, Italie méridionale, terre cuite polychrome, 54,8 × $16 \mathrm{~cm}$ (gauche) et 56,3 × $15 \mathrm{~cm}$ (droites), Lyon, musée des beaux-arts, E 406-3-a et E 406-3-b.

(c) Lyon MBA - Photo Alain Basset.

9 Les terres cuites, l'ouvrage majeur de Biardot, se donnait pour but d'expliquer le symbolisme des terres cuites. En effet, pour notre antiquaire, elles sont toutes liées aux mystères de Bacchus et il est possible de les interpréter finement en se fondant sur leur iconographie et sur des textes anciens ainsi que sur des travaux contemporains comme ceux de Friedrich Creuzer ${ }^{50}$, d'Alfred Maury ${ }^{51}$, ou encore de Bachofen. Biardot se livre ainsi à une exégèse complexe dont il anticipe la critique en écrivant: «l'induction accompagnée d'une certaine hardiesse, la divination dans une certaine mesure semblent bien permises quand les documents sont si rares et si timides. Il s'agit moins ici, après tout, de l'interprétation irréfragable de chaque fait que de la clef de l'intelligence générale de ce monde mystérieux; d'en faire sentir le concert, la symétrie, les courants divers mais concentriques $»^{52}$. Outre un phrasé pompeux et l'emploi de termes à connotation scientifique, sa démarche semble s'appuyer, comme il se doit sur de nombreuses sources littéraires, le plus souvent citées en notes de bas de page, mais on voit d'emblée qu'elles n'ont pas été harmonisées. Ainsi, Biardot utilise des formes abrégées des noms d'auteurs, diversement citées selon les notes. La multiplicité des abréviations laisse penser que notre auteur ne fait pas forcément le rapprochement entre «Plutarque », «Plutarq.» et «Plut.» ni, encore moins, entre "Cornutus» et «Phurnut. ${ }^{53}$. De même, les titres des ouvrages sont mentionnés parfois en français et d'autres fois en latin. Ce manque d'uniformité nous a donc amenés à approfondir l'analyse. En les entrant dans le moteur de recherche Google Livres, il est apparu qu'un certain nombre d'entre elles sont directement tirées d'ouvrages que Biardot a pu consulter. Nous considérons ici que deux notes de bas de 
page de deux ouvrages différents correspondent si leur texte est identique et si la proposition qu'elles viennent étayer est la même. Ainsi, la note de la page 238 " Aristoph., Acharn., 239 " sert à étayer l'hypothèse suivante : «En poursuivant cette idée, nous trouvons encore dans les Dionysies exotériques, parmi les offrandes destinées au héros de la fête, les figues que portaient dans des corbeilles d'or de jeunes vierges nubiles remplissant les fonctions de Canéphores ${ }^{54}$. Et dans la première partie du troisième volume des Religions de l'antiquité considérées principalement dans leurs formes symboliques et mythologiques de Joseph Daniel Guigniaut, on trouve page 229 la note « Aristoph., Acharn., 239 » qui justifie l'idée : « Entre autres offrandes destinées au dieu, des figues sont citées, que portaient dans des corbeilles d'or des vierges nubiles, nommées pour cette raison Canéphores ». Cette correspondance n'est pas isolée. Au total, nous avons établi que 115 notes de bas de page sur les 277 recensées proviennent d'autres ouvrages ${ }^{55}$. Ces plagiats se concentrent, sans surprise, dans les chapitres où Biardot développe le plus longuement ses théories, contrairement à ceux qui relatent son expérience de collectionneur ou dans le chapitre introductif. Les terres cuites ressemble ainsi plus à un travail de compilation qu'à une réflexion originale, ce qui n'est finalement guère étonnant au regard de son absence de formation initiale. Nous avons même pu retrouver une lettre de Biardot dans laquelle il demande à Bachofen l'autorisation de le plagier ${ }^{56}$, preuve que ces emprunts ne sont pas innocents et que notre antiquaire a conscience de recourir à une pratique proscrite dans les milieux scientifiques.

Fig. 4

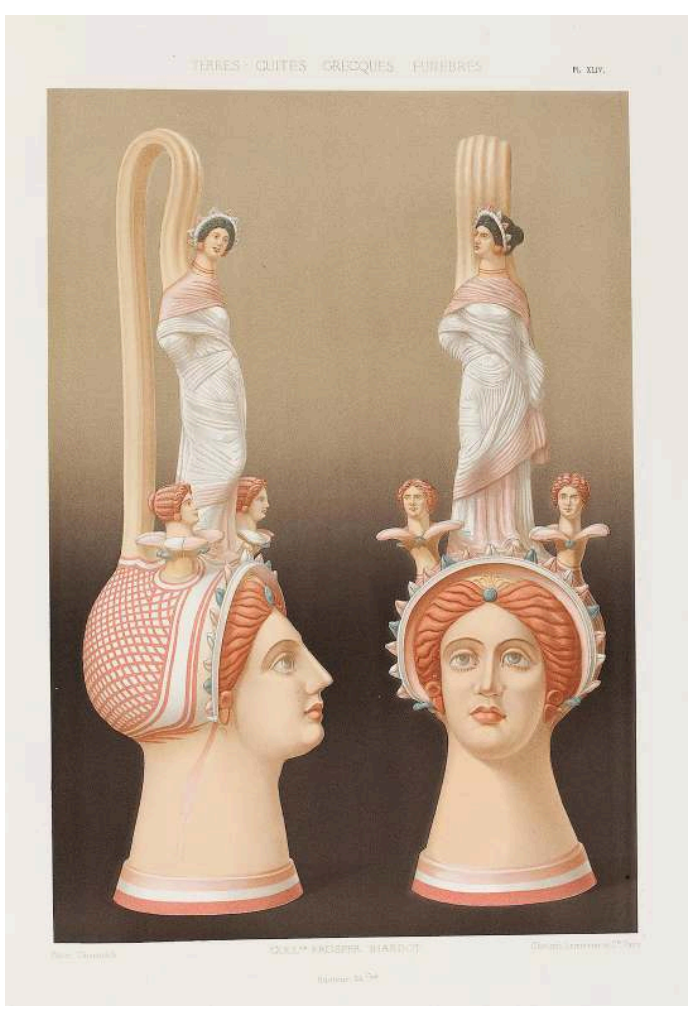

Biardot, Prosper, Atlas, planche XLIV : vase plastique en forme de tête féminine. Heidelberg University Library 


\section{Réception de ses ouvrages}

10 L'Explication était déjà, de l'aveu même de son auteur, passée relativement inaperçue en France. Il écrit ainsi dans l'introduction de son ouvrage de 1872: «J'envoyais ma brochure à tous ceux que leur science, à tous ceux que leur savoir-faire scientifique ont établi en France les juges naturels ou obligatoires des archéologues nouveau-venus (sic). J'eus la simplicité de croire qu'ils m'en diraient leur avis, à tout le moins qu'ils le diraient au public. J'attends encore; j'attendrai peut-être toujours $»^{57}$. On remarque toutefois un article de Fröhner publié dans la Revue contemporaine qui accable notre antiquaire de manière cruellement ironique en l'accusant de se livrer à la fougue de son imagination plutôt que de recourir à la rigueur scientifique : «Il faut en convenir, la délectation d'interpréter jusqu'aux choses les plus insignifiantes n'est pas donnée à tout le monde, et là ou nous autres antiquaires n'éprouvons rien, M. Biardot doit ressentir des plaisirs intimes, des voluptés secrètes qu'on pourrait lui envier $\aleph^{58}$. En revanche, des érudits étrangers, notamment germaniques dans un contexte politique tendu entre la France et l'Allemagne, s'étaient montrés plus réceptifs. Gerhard, bien que relativement circonspect, en avait publié un compte rendu de lecture dans l'Archäologischer Anzeiger de juillet 1864. D'autres savants allemands avaient écrit à Biardot qui leur avait envoyé son ouvrage, afin, que selon notre auteur, de l'encourager dans ses recherches ${ }^{59}$. L'accueil de l'ouvrage de 1872 ne diffère pas. Mis à part un article prudent dans la Revue archéologique $e^{60}$, les réactions des savants sont plutôt horrifiées par les thèses présentées. Ainsi Edmond Pottier écrit-il : «Cette thèse a été présentée sous une forme tellement outrée, et, en quelque sorte, apocalyptique, qu'elle échappe par sa nature même à la discussion. Aucun texte ancien, aucun document sérieux n'est invoqué à l'appui des explications données $»^{61}$. Aujourd'hui, ces théories sur le symbolisme des terres cuites sont largement dépassées et oubliées. C'est finalement le volume de planches qui connaît le plus de succès. Les dessins luxueux commandés par Biardot sont repris dans diverses publications sur les terres cuites ${ }^{62}$ et fournissent des éléments intéressants pour les études sur la polychromie des figurines, malgré les exagérations évidentes, ainsi que sa perception au XIX ${ }^{e}$ siècle $^{63}$.

11 Biardot, personnage tout en ambiguïtés, dont la vie reste mal connue, conserve son mystère, comme demeure trouble le rôle qu'a pu y jouer Amelia Giovanna O'Donovan Hamilton. D'elle, d'ailleurs, rien, ou presque ne subsiste même pas une tombe puisque, puisque dans son testament ${ }^{64}$, elle demande à être enterrée en fosse commune. Doit-on reconnaître sa patte dans la démarche de collectionneur de Biardot? Napolitaine (elle arrive en tout cas à Naples avant notre archéologue), elle semble plus imprégnée de culture classique que lui, que rien ne prédispose à s'intéresser à l'archéologie. C'est probablement à elle, d'ailleurs, que Fröhner pense quand il évoque les idées de " Madame Prosper Biardot $"{ }^{65}$. Paradoxalement, Biardot a failli s'effacer derrière sa compagne dans les bibliographies modernes, qui le tiennent, pour la plupart, pour une femme.

Sa collection, constituée à Naples, nous est elle aussi méconnue. Le catalogue de la vente de 1874 n'a en effet pas été conservé et les autres catalogues dont nous disposons sont soit trop imprécis, dans le cas de l'inventaire après décès, soit non exhaustifs dans les cas de l'Atlas ou du catalogue manuscrit conservé à l'INHA. Finalement, les renseignements que nous avons retrouvés, en soulevant plus de questions qu'ils n'apportent de réponses, forment une sorte de portrait en creux de notre 
collectionneur. Combien d'objets de la collection Campana du Louvre Biardot a-t-il fait reproduire en les mêlant à ses propres œuvres? Sa collection contenait-elle également des moulages et en quelle proportion? La fausse argenterie pose enfin la question de l'honnêteté de notre antiquaire. Ses réactions contradictoires et sa violente altercation avec Longpérier ${ }^{66}$ laissent penser que, s'il s'était laissé duper, il était prêt à en tromper d'autres.

Ses ouvrages font preuve, là encore, d'ambiguïté. Biardot rêvait probablement de marquer l'histoire de l'archéologie en rédigeant un des premiers livres consacrés aux figurines de terre cuite et ainsi prouver son érudition afin d'intégrer un milieu social intellectuel qui n'était pas le sien, mais l'analyse de ses sources a montré ses lacunes. Manifestement, ses théories sur le symbolisme n'ont pas été acceptées par ses contemporains et relèvent aujourd'hui tout au plus de la curiosité. C'est finalement l' Atlas, surtout pour la qualité de ses illustrations, et, de façon ironique et paradoxale, l'ignorance touchant au nom même de Prosper Biardot, qui ont permis de conserver le souvenir de cet homme et de sa collection.

\section{NOTES}

1. www.bibliotheque-numerique.inha.fr/collection/item/16545-catalogue-des-objets-dart-antiques-de-la-collection-prosper-biardot?offset=1" $\backslash \mathrm{h}$ Manuscrit de la collection d'orfèvrerie ayant appartenu à Prosper Biardot [12/02/2019].

2. www.idref.fr/095810781 Notice d'Elizabeth Caroline Hamilton Gray [12/02/2019].

3. Wilhelm Fröhner, Terres cuites d'Asie de la collection Julien Gréau, 1886, Paris, H. Hoffmann, p. X.

4. Allemagne, Weimar, Klassik Stiftung, Goethe- und Schiller Archivs: Fröhner, Wilhelm, Eingegangene Briefe. Dans la suite de cet article, nous désignerons ce fonds par « Archives Wilhelm Fröhner ».

5. Suisse, Bâle, Bibliothèque de l'université de Bâle : Fonds Johann Jakob Bachofen, NL $3: 272,11-20$. Dans la suite de cet article, nous désignerons ce fonds par «Archives J. J. Bachofen ».

6. Archives de Paris, actes d'état civil reconstitués, 5 Mi 1/129.

7. Nous ignorons les raisons qui motivent ce voyage.

8. Archives nationales, Ministère de l'Intérieur - Police générale, délivrance de passeports intérieurs et extérieurs: enregistrements, MIC/F/7/2568: demande du 11 mai 1835.

9. Archives Wilhelm Fröhner, Signatur GSA 204 : notice de Wilhelm Fröhner sur les épouses de Prosper Biardot.

10. Cat. d'exp., Un rêve d'Italie, la collection du marquis Campana, sous la direction de Françoise Gaultier, Laurent Haumesser, Anna Trofimova, Paris, Musée du Louvre, nov. 2018 - fév. 2019, Liénart et Musée du Louvre éditions, et notamment pp. 90-117. 
11. Adrien de Longpérier (Paris 1816 - Paris 1882) : archéologue et conservateur au Cabinet des Médailles puis au musée du Louvre.

12. Wilhelm Fröhner (Karlsruhe 1834 - Paris 1925) : collectionneur et conservateur du Louvre jusqu'en 1870 où il perd son poste du fait d'accusations d'espionnage. Par la suite il vit des rédactions de catalogues d'antiquités.

13. Salomon Reinach, « Notice biographique » dans Olivier Rayet, Études d'archéologie et d'art, 1888, Paris, librairie Firmin Didot et Cie, p. X.

14. Violaine Jeammet, «Un certain goût pour les Tanagras » dans Violaine Jeammet, Tanagras. De l'objet de collection à l'objet archéologique, 2007, Paris, Musée du Louvre éditions, p. 40.

15. Léon Heuzey en 1874 note que les théories de Prosper Biardot sont sans valeur dans "Recherches sur les figures de femmes voilées dans l'art grec », Monuments grecs publiés par l'association pour l'encouragement des études grecques en France, $\mathrm{n}^{\circ}$ 3, p. 13.

16. Johann Jakob Bachofen (Bâle 1815 - Bâle 1887) : juriste, philologue. Auteur de Das Mutterrecht en 1861, il cherche dans les mythes les traces d'un matriarcat originel, qui aurait donné lieu ensuite au patriarcat.

17. Johann Jakob Bachofen, Gesammelte Werke / Johann Jakob Bachofen, hergestellts von Karl Meuli, X, 1943, Bâle, B. Schwabe, pp. 326-331. Texte original: "Solche Leute, die keiner Clique angehören und keine bediensteten Professoren sind, dagegen Jahre lang an Ort und Stelle nur der Sache leben, sind mir besonders werthvoll ». Traduction de l'auteur.

18. Titre original: Die Unsterblichkeitslehre der orphischen Theologie auf den Grabdenkmälern des Altertums: nach einer Anleitung einer Vase aus Canosa im Besitz des Herrn Prosper Biardot in Paris. Traduction de l'auteur.

19. Archives nationales, Minutier central, MC/DC/LVIII/899, document du 11 juillet 1873.

20. www.bibliotheque-numerique.inha.fr/idurl/1/16545 "Catalogue des objets d'art antiques de la collection Prosper Biardot ", vers 1874, manuscrit par Raymond Dournès, inv. MS 741 [05/02/2019].

21. Cat. d'exp., Principi, imperatori, vescovi, sous la dir. de Raffaella Cassano, «Ipogei Lagrasta », Bari, monastère Santa Scolastica, janvier-mai 1992, Venise, Marsilio, 1992, pp. 203-224.

22. Prosper Biardot, Les terres cuites grecques funèbres dans leur rapport avec les mystères de Bacchus, 1872, Paris, Firmin Didot frères. Dans la suite de l'article, cet ouvrage sera désigné par Les terres cuites.

23. Prosper Biardot, Les terres cuites grecques funèbres dans leur rapport avec les mystères de Bacchus: l'auteur a ajouté en faveur des artistes une série de figurines drapées tirées de sa collection et de l'ancien cabinet de M. le vicomte de Janzé, 1872, Paris, Firmin Didot frères. Disponible en ligne www.digi.ub.uni-heidelberg.de/diglit/biardot1872bd2 [05/02/2019]. Dans la suite de l'article, cet ouvrage sera désigné par L'Atlas.

24. Lancelot Théodore Turpin de Crissé (Paris 1782 - Paris 1859): peintre et collectionneur d'art.

25. Lancelot Théodore Turpin de Crissé, «Lettre à Monsieur le directeur du Musée d'Angers, Paris, février 1858 " dans Henry Jouin, Les maîtres peints par eux-mêmes, sculpteurs, peintres, architectes, musiciens, artistes dramatiques, 1902, Paris, GaultierMagnier, p. 360. 
26. Biardot, Les terres cuites, p. 551.

27. Idem, Ibid., p. 407.

28. Id., Ibid., p. 407.

29. Archives de Paris, État civil, Actes de décès du $6^{\mathrm{e}}$ arrondissement, V4E 3148, acte $\mathrm{n}^{\circ} 1186$ : acte de décès du 4 juin 1873.

30. Thérèse Diebolt (1848 - 1907) : en 1872, elle vit à Paris où elle travaille comme domestique et a deux enfants naturels. Elle épouse Prosper Biardot en 1872. Après son décès en 1873, elle épouse Jean-Michel Kapp, le père de ses enfants, en 1874.

31. J.G., "Vente Biardot - Terres cuites grecques funèbres ", L'indicateur de l'archéologue et du collectionneur : bulletin mensuel illustré, $\mathrm{n}^{\circ} 15$, mars 1874, pp. 158-160.

32. La vente à Ed. de Verdier et le procès sont documentés par la Note en réponse au dispositif d'un jugement rendu par le Tribunal Civil de la Seine le 18 juin 1898 entre les Époux Kapp et M. de Verdier et autres et Conclusions motivées pour M. de Verdier contre M. et Mme Kapp, les héritiers Dournès et M. Daubresse. Archives Wilhelm Fröhner, Signature GSA $107 / 121$.

33. Wilhelm Fröhner, Collection J. Gréau. Troisième partie. Terres cuites grecques, vases peints et marbres antiques, 1891, Paris, H. Hoffmann.

34. AP, Archives judiciaires, Étude de maîtres Delbergue, Delestre, Desvouges et Boisgirard : minutes et dossiers de ventes, 1851-1969, 2 février 1891 - 31 octobre 1891 : D60 E3 56.

35. Il s'agit de la figurine CA 409.

36. Il s'agit des vases V.I. 3195 et V.I. 3196. Malheureusement, ces deux canthares ont été détruits par des bombardements pendant la Seconde Guerre mondiale. Martin Miller, Ursula Kästner, Dokumentation der Verluste V: Antikensammlung, 2005, Berlin, Staatliche Museen zu Berlin - Preußischer Kulturbesitz, p. 170.

37. Il s'agit du lécythe plastique inv. 3737.

38. Ce sont les vases E 406-3-a et E 406-3-b.

39. Wilhelm Fröhner, Collections du Château de Gołuchów, Antiquités, décrites par W. Froehner: Objets égyptiens, vases peints (supplément), terres cuites, verrerie, bronzes, poids antiques, ivoires et os, marbres, antiquités de Carthage, 1899, Paris, imprimerie alsacienne.

40. Il s'agit du vase 142264/1-2 MNW.

41. Il s'agit de l'œnochoé inv. 1254.

42. Wilhelm Fröhner, Collection J. Gréau. Troisième partie. Terres cuites grecques, vases peints et marbres antiques, 1891, $\mathrm{n}^{\circ} 134$, p. 38. Cat. d'exp., Bourdelle et l'Antique, sous la direction de Claire Barbillon, Jérôme Godeau, Amélie Simier, Musée Bourdelle, oct. 2017-fév. 2018, Paris, Paris-Musées, 2017, p. 46, cat. 8. Jeammet, Violaine "De l'intuition au palimpseste, la Grèce fantasmée de Bourdelle", in Barbillon, Claire, Godeau Jérôme, SImier, Amélie (dir.) Bourdelle et l'antique une passion moderne, [exposition, Paris, musée Bourdelle, 4 octobre 2017 - 4 février 2018], 2017, pp. 36-53 (au moment de cette publication, l'historique n'avait pas été encore tracé).

43. Sébastien Norblin (Varsovie 1796 - Paris 1884) : peintre. Titulaire du prix de Rome en 1825 il séjourne six ans en Italie où il commence à constituer une collection d'objets d'art (tableaux, dessins, gravures et antiquités, notamment une collection égyptologique). 
44. An, Archives de l'École Nationale Supérieure des Beaux-Arts, Liasses, Collections, Collections du Musée des études et de la Bibliothèque : dons et legs, $\mathrm{xIX}^{\mathrm{e}}-\mathrm{xx}^{\mathrm{e}}$ siècles, Dossiers alphabétiques des donateurs composés de correspondance et d'inventaire des collections, AJ/52/449 : H-O.

45. Prosper Biardot, op. cit., p. V.

46. Prosper Biardot, Explication du symbolisme des terres cuites, 1864, Paris, librairie de Humbert. Dans la suite de l'article, cet ouvrage sera désigné par Explication.

47. Prosper Biardot, op. cit., 1872, p. 362.

48. Idem, Ibid., p. 419.

49. Id., Ibid., p. 429.

50. Friedrich Creuzer (Marbourg 1771 - Heidelberg 1858) : archéologue, philologue. Il est l'auteur de La symbolique et la mythologie des peuples anciens publié entre 1810 et 1812 . Cet ouvrage a été largement diffusé par Joseph-Daniel Guigniaut qui l'a traduit et enrichi.

51. Alfred Maury (Meaux 1817 - Paris 1892) : archéologue, historien, professeur, il publie Histoire des religions de la Grèce antique de 1857 à 1859.

52. Prosper Biardot, op. cit., 1872, pp. 52-53.

53. Lucius Annaeus Cornutus est également connu sous le nom de Phurnutus. Voir Data.bnf.fr qui indique les variantes du nom:www.data.bnf.fr/12568858/ lucius_annaeus_cornutus [05/02/2019].

54. Ibid.

55. Il faut en outre tenir compte que nous avons été dépendante pour cet exercice de l'océrisation (procédé informatique qui permet la traduction d'images de textes imprimés ou dactylographies en fichiers de texte) proposée par Google Livres. D'autres sources de Prosper Biardot ont donc pu nous échapper, faute de numérisation ou faute d'océrisation.

56. Archives J. J. Bachofen, NL 3: 272, 18: lettre de Prosper Biardot datée du 12 décembre 1867.

57. Prosper Biardot, Les terres cuites, pp. V-VI.

58. Wilhelm Fröhner, "Travaux des académies et des sociétés savantes: sciences historiques et archéologiques", Revue contemporaine, $2^{e}$ série, tome 39, 1864, Paris: Bureaux de la revue contemporaine, pp. 376-377. Nous ne connaissons pas de réaction, quelle qu'elle soit, de Prosper Biardot dont les relations avec Wilhelm Fröhner semblent s'être interrompues brutalement en 1860.

59. Prosper Biardot, op. cit., 1872, pp. VI-XI.

60. «Les terres cuites », Revue archéologique, volume XIV, 1873, pp. 437-439.

61. Edmond Pottier, Les statuettes de terre cuite dans l'antiquité, 1890, Paris, librairie Hachette et Cie, p. 265.

62. Parmi ces publications : Edmond Pottier, op. cit., fig. 75 p. 215 et Franz Winter, Die antiken Terrakotten im Auftrag des archäologisches Instituts des deutschen Reichs. Band III : die Typen der figürlichen Terrakotten, 1903, Berlin et Stuttgart: Verlag von W. Spemann, pp. 129, 131, 148, 151, 152, 171, 205. Voir aussi Cassano, cat. exp. cité plus haut.

63. Cat. d'exp., En couleurs, la sculpture polychrome en France 1850-1910, sous la direction d'Édouard Papet, «Les paradoxes de l'invention de la polychromie antique au XIXe » de 
Brigitte Bourgeois, Violaine Jeammet, Paris, musée d'Orsay, juin-septembre 2018, Paris, Hazan, pp. 151-156.

64. An, Minutier central, MC/ET/LVIII/887 document du 5 novembre 1870.

65. Wilhelm Fröhner, op. cit., 1886, p. X.

66. BnF, Département des Monnaies, médailles et antiques, Fonds de la correspondance de Raoul-Rochette, 1 APM (2004-271) : 2004-271-329 : lettre d'Adrien de Longpérier à Désiré Raoul-Rochette datée du 15 juillet 1852.

\section{RÉSUMÉS}

Entre un antiquaire féru d'archéologie napolitaine et un imposteur, la figure de Prosper Biardot se révèle paradoxale à bien des égards: sa collection se compose de terres cuites particulièrement intéressantes provenant de Grande Grèce, et, pour un certain nombre d'entre elles notamment, d'un des premiers hypogées fouillés à Canosa (Pouilles), dit Lagrasta I. Il se fit pourtant une réputation peu flatteuse dans le milieu archéologique français en raison de ses achats inconséquents dans le domaine de l'orfèvrerie, et de ses idées, alors qu'il trouve un certain écho à l'étranger, en particulier en Allemagne, et surtout en Suisse auprès du philologue Johann Jakob Bachofen. Ses publications rassemblent en effet des idées contrastées oscillant entre des positions très novatrices sur les restaurations et des théories largement dépassées aujourd'hui sur le symbolisme des terres cuites, qui s'inscrivent dans le courant de l'époque. Son album de planches chromolithographiées est l'un des rares témoignages illustrés de ce type d'œuvres, et à ce titre régulièrement cité dans l'historiographie de la discipline. C'est le parcours et l'identité de cette personnalité ambiguë $d u$ collectionnisme du xixe siècle, contemporaine du marquis Campana, que cet article se propose d'éclairer tandis que l'analyse des sources de ses ouvrages permet de mieux comprendre le travail mené par cet amateur.

Somewhere between an antiquarian keen on Neapolitan archaeology and an impostor, Prosper Biardot cut a paradoxical figure in many ways: his collection comprised particularly interesting terracottas from Magna Graecia and, for some of them, one of the first hypogea excavated in Canosa, Puglia, known as Lagrasta I. He made a not terribly flattering reputation for himself in French archaeological circles because of his inconsistent purchases in the domain of gold- and silverwork and his ideas, which found an echo abroad, particularly in Germany, and especially Switzerland in the thinking of philologist Johann Jakob Bachofen. His publications contain contrasting ideas fluctuating between very innovative positions on restorations and now outdated theories on the symbolism of the terracottas, which were part of the trends of the times. His album of chromolithographic plates is, on the other hand, one of the rare illustrated accounts of this type of work and for this reason regularly cited in the historiography of the discipline. It is the career and the identity of this ambiguous personally of nineteenth-century collecting, the contemporary of Marquis Campana, that this article sets out to illuminate while the analysis of the sources of his books allow us to understand better his work. 


\section{INDEX}

Mots-clés : Biardot, terre cuite, Naples, collectionneur, XIXe siècle, archéologie, faux archéologique, plagiat, chromolithographie

Keywords : Biardot, terracotta, Naples, collector, nineteenth century, archaeology, archaeological fake, plagiarism, chromolithography

\section{AUTEUR}

\section{ANGÉLIQUE ALLAIRE}

Angélique Allaire est diplômée du deuxième cycle de l'École du Louvre (parcours "recherche" en histoire de l'art) et de l'ESSEC Business School (Master in Science of Management). Elle a soutenu en 2018 un mémoire de recherche, sous la direction de Violaine Jeammet et Corinne Jouys Barbelin, consacré au collectionneur Prosper Biardot.

Angélique Allaire is a PhD student at Sorbonne Université (Centre André Chastel UMR 8150 and Labex OBVIL). She is a graduate of the École du Louvre (research master, art history) and the ESSEC Business School (MSc in Management). In 2018 she defended a research thesis on the collector Prosper Biardot, which was supervised by Violaine Jeammet and Corinne Jouys Barbelin. 